\title{
Monitoring for Nutrition Results in ICDS: Translating Vision into Action
}

\author{
Saroj K. Adhikari and Caryn Bredenkamp
}

\begin{abstract}
This article focuses on the Integrated Child Development Services (ICDS), India's largest nutrition and early child development programme. It describes the political, organisational and technical challenges to building and sustaining an outcomes-oriented approach to nutrition monitoring in India. We show that the environment is conducive to strengthening nutrition programme monitoring and evaluation. Political commitment is growing, financial allocations have increased and there have been a number of reforms to strengthen the ICDS monitoring systems, but weaknesses remain. The article analyses seven technical challenges to improving the outcomes-orientation of ICDS and suggests steps that could be taken to improve monitoring.
\end{abstract}

\section{Introduction}

The Integrated Child Development Services (ICDS) programme is India's primary response to the nutritional and developmental needs of children under six years, pregnant women and nursing mothers. Implemented through a network of over 1 million village-level Anganwadi Centres (AWCs), staffed by Anganwadi Workers (AWWs) and Anganwadi Helpers (AWHs), it currently reaches around 70 million children and about 15 million pregnant and nursing mothers (MWCD 2009).

For over three decades, despite shifts in political power, the Government of India (GoI) has continued to expand ICDS, increasing the financial allocation to the programme with each successive five-year development plan. In 2001, following a landmark Supreme Court order that directed central and State Governments to 'implement the ICDS in full' and ensure that every AWC provided children with specified minimum quantities of food, Government support to ICDS suddenly rose sharply (MWCD 2006). The budgetary allocation to ICDS increased more than four-fold from Rs.10,392 crore ( 1 crore $=10$ million rupees) (US $\$ 2.2$ billion) for the Tenth Five Year Plan (2002/07) to Rs.44,400 crore (US $\$ 9.45$ billion) for the current Eleventh Plan (2007/12). These funds are intended to support the 'universalisation' of ICDS and the attainment of the exceptionally ambitious target of halving the current prevalence of malnutrition by 2012 .

The budget increase signals Government commitment to improving the nutritional status of children. It also puts pressure on policymakers and implementers to show that the financial investments are yielding results. If ICDS is to reduce child malnutrition, programme managers need to have a reliable and efficient monitoring system to measure progress and adjust programme implementation in order to achieve the desired results. We explore the emergence of an outcomes-oriented approach to nutrition programme implementation and monitoring, as articulated in recent political statements and evidenced in policy reforms. We also examine challenges to improving the outcomesorientation of ICDS and identify steps that can be taken to strengthen the focus on results.

\section{What is an outcomes-oriented approach to alleviating malnutrition and how can it help?} Effective monitoring is about collecting the right data at the right time and at the right level of measurement. It is also about using the data to improve programme management, decisionmaking and implementation. This shifts the 
Figure 1 An outcomes-oriented approach to nutrition monitoring

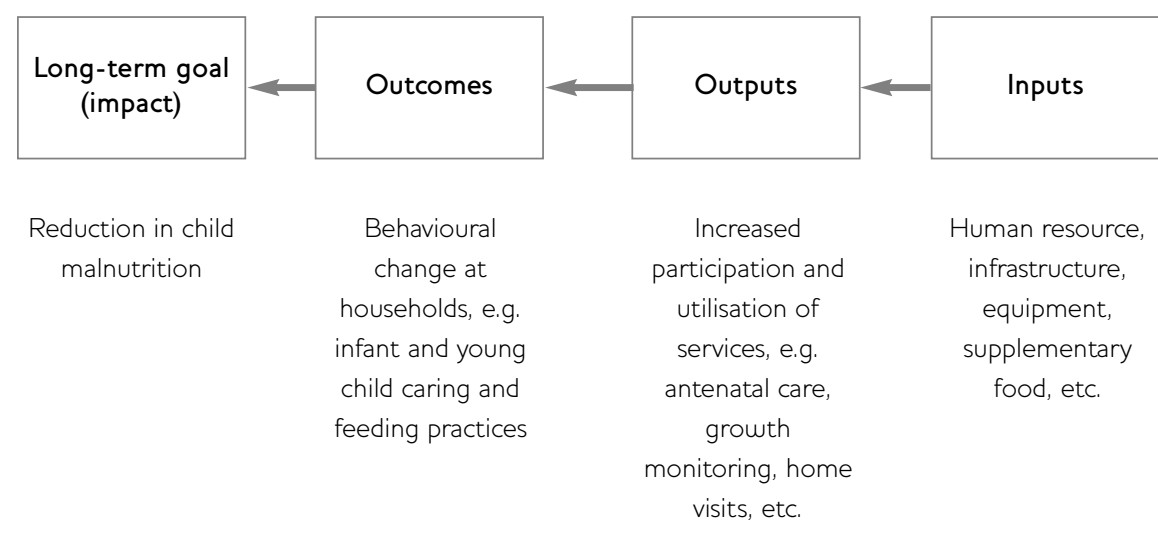

Results

Implementation

Traditional M\&E

focus from inputs to outputs and outcomes, focusing not only on expenditures but rather on what is achieved with those expenditures.

Collecting the 'right data' means measuring indicators along the entire results chain - inputs, outputs, outcomes and impact (Figure 1). In most government programmes, the focus tends to be on collecting data on inputs, such as expenditure, staffing, training and equipment. However, this approach does not reveal what these investments yield in terms of the quality and quantity of services delivered, and the effect of service delivery on programme outcomes. Consequently, programme managers do not have the information they need to assess whether the programme is reaching its objectives and whether corrective action is required.

An outcomes-oriented approach demands a shift from measuring inputs and outputs ('traditional $\left.\mathrm{M} \& \mathrm{E}^{\prime}\right)$ to measuring results at the level of outcomes. The starting point should be to identify what the programme aims to achieve - the desired impact and outcomes of the programme and then work 'backwards' to determine which outputs are needed to attain those outcomes, and, in turn, the inputs that are needed to deliver those outputs. We emphasise this by drawing the results chain in 'reverse'. In a nutrition programme, with a long-term goal of reducing the prevalence of child malnutrition, an outcomesorientation means focusing on behavioural changes (such as infant and child feeding and caring practices) at the household levels and, based on the outcomes observed, selecting the type, quality and quantity of health-promoting services (such as antenatal care, growth monitoring or home visits) to be delivered, and then deciding what inputs/resources are needed to deliver those outputs.

\section{Political commitment: a window of opportunity?}

Building a results-based M\&E system is not easy and once built, the challenge is to sustain it. While there are many organisational, and technical challenges to be overcome, building and sustaining such a system is primarily a political process, and less so a technical one (Kusek and Rist 2004). It requires institutional reforms from the highest to the lowest implementation levels. This will be possible only if there is strong political commitment both at national and state levels - and if commitment moves beyond vision to action and follow-up action.

In recent years, a notable commitment to an outcomes-oriented approach to social sector programmes, including nutrition, has been expressed at the highest levels. A watershed 
moment was the development of the GoI's first 'outcome budget' in 2005, setting out a clear agenda for focusing on outcomes. On 28 February 2005, while presenting the budget to Parliament, the Finance Minister of India cautioned:

... outlays did not necessarily mean outcomes ... We shall also ensure that programmes and schemes are not allowed to continue indefinitely ... without an independent and in-depth evaluation ...

(MoF 2005)

This results-orientation is also evident in the Eleventh Plan (2007/12). It states:

It is especially important to improve evaluation of the effectiveness of how Government programmes work and to inject a commitment to change their designs in the light of the experience gained. Evaluation must be based on proper benchmarks and be scientifically designed to generate evidence-based assessment of different aspects of programme design.

(Planning Commission 2008)

In 2005/06, the Ministry of Women and Child Development (MWCD) also adopted an 'outcome-budget', including financial data, intermediary processes and activities. While a noteworthy step in the right direction, this budget needs to be supported by a clear implementation plan and time-line. This is also critical to implementing the Prime Minister's vision, articulated in his letter to State Chief Ministers (9 January 2007), that 'proper implementation of the programme critically depends on political will, decentralised monitoring and meticulous attention to day-today operational issues'.

4 Technical challenges in adopting an outcomesoriented approach within the ICDS

In a programme as large and complex as ICDS, building an effective outcomes-orientation is difficult and there are many technical challenges.

\subsection{Shifting from a 'monitoring' information system to a 'management' information system}

Although ICDS is a 'centrally sponsored scheme', and the GoI prescribes a standardised monitoring process, primary responsibility for programme implementation and monitoring rests with the State Governments. Every month, data are collected by the AWWs from over 1 million AWGs. These data are aggregated as monthly progress reports (MPRs) and transmitted to higher levels of programme management. The central ICDS monitoring cell analyses data on a limited number of indicators (viz. operationalisation of Blocks/AWCs, staffing positions, beneficiaries of supplementary food and pre-school education, and nutritional status of children) and sends feedback on progress to the States.

While the ICDS monitoring information system (MIS) has been in place for many years, its use as an effective tool for improving programme implementation has been limited. What is absent (except in a few States) is a systematic analysis of relevant indicators at State, District and Block levels and a feedback mechanism to inform field functionaries about the programme's effectiveness. This means that local action is seldom taken in response to the information that is generated at AWCs. External monitoring or random checks of data quality are rare. Thus, although the programme is monitored - in the sense that information on inputs and outputs is collected - the system is not oriented toward using that information to inform action (Gragnolati et al. 2006).

\subsection{Overcoming human resource and capacity constraints}

The ICDS MIS entrusts all of its data collection to the AWW, an honorary worker who has limited capacity to use the data to manage and improve service delivery. Supervisors and CDPOs are often educated to university level and have clearly defined monitoring and supervision roles, but are not always sufficiently trained in monitoring. The MIS is discussed during mandatory job training, but only 3-4 per cent of training time is devoted to it - six hours for AWWs and eight hours for their Supervisors (NIPCCD 2006). Moreover, training tends to be limited to how to fill in registers/MPRs rather than strengthening competencies in the validation, interpretation, analysis or use of data for decision-making.

Staff shortages impose additional constraints. Although ICDS has expanded rapidly in recent years and created new field level positions, the existing staffing pattern does not provide specialist monitoring positions at the State/District levels, leaving the task mainly to administrative personnel with little technical 
knowledge. The number of qualified people assigned to monitoring is relatively small and staff turnover is high. At field level, some 31 per cent of Supervisor positions and 34 per cent of CDPO positions are vacant (MWCD 2009).

\subsection{Defining outcomes for measuring ICDS performance} Through the routine MIS, the ICDS programme tracks inputs, some intermediary processes and limited outputs, rather than nutrition outcomes such as appropriate infant care and feeding behaviours.

While the collection of input, process and output indicators are important for programme management, they are insufficient to measure programme performance. Indicators that are further up the results chain, such as the take-up rates of services, or at the level of outcomes, such as the number of mothers adopting appropriate feeding behaviours, need to be monitored to help strategise programme inputs/processes.

However, the measurement of outcomes is only possible through periodic household surveys and, so, for short-term monitoring, and until strategies for regular outcome measurement at the household level are developed, the MIS will need to focus on output indicators.

The MIS also tracks one impact indicator, namely the nutritional status of children. While the measurement of nutritional status is important for (i) local-level growth promotion, and (ii) evaluation of programme impact over the longer-term, it is not a pragmatic measure for programme management or a realistic measure of ICDS performance. First, experience suggests that, at the population level, even 'short route interventions', such as growth promotion for young children, low birth weight prevention programmes, micronutrient programmes and food assistance programmes (Shekar et al. 2006), take a number of years to show results. Second, nutritional status is multi-causal and the result of a complex interaction of immediate, underlying and basic causes (UNICEF 1998), many of which lie outside the realm of influence of ICDS, making it difficult to attribute changes in nutritional status to ICDS implementation. Rather than using nutritional status as the major measure of programme performance, the MIS should focus on the output and outcome indicators that help officials to manage the programme.

\subsection{Ensuring sectoral convergence in monitoring}

While the reduction of child malnutrition is one of the core objectives of ICDS, the underlying causes of malnutrition are multidimensional and the roles of other sectors, especially health, education, sanitation, water and rural development, in reducing malnutrition are essential and well established. Yet, the administrative division of responsibility puts the onus of reducing malnutrition exclusively on the MWCD, which is mandated to provide nutrition and health education and supplementary nutrition services through ICDS. The ICDS MIS is the only mechanism that the GoI has in place to regularly monitor malnutrition, but since it only monitors those services provided by ICDS, it provides incomplete information about the causes of malnutrition.

Malnutrition requires a multi-sectoral response with a common multi-sectoral results framework for malnutrition, an implementation plan that includes indicators and targets for all related departments and periodic joint reviews on progress. Convergent monitoring would help to bring about convergence in effort and responsibility.

\subsection{Prioritising services to measure}

Ironically, effective monitoring in the ICDS programme is inhibited by too much data collection. The programme delivers six different services targeted at different groups (0-6 year-old children, pregnant women and nursing mothers), which means that there are a number of outcomes that could be monitored. Realistically, quality monitoring is only possible if a few outcomes are measured and careful thought, taking into consideration the local context, is given to which outcomes these should be.

In ICDS, indicators related to the supplementary nutrition programme (SNP) tend to be prioritised because this is the only intervention that is directly linked to a financial allocation (Rs.4/child per day); the number of beneficiaries determines the amount of money to be allocated to food procurement. The result is that, compared with other nutrition services, records for supplementary food are meticulously maintained and checked by the AWWs and Supervisors. Indeed, the success of the programme is often reported on the basis of the increase in the number of SNP beneficiaries. 
This is one area where the programme has succeeded in establishing an effective monitoring system. However, nutrition is about more than food supplementation, and an over-emphasis on SNP indicators often leads to the neglect of other key nutrition services such as nutrition and health education, counselling of pregnant and nursing mothers, growth promotion etc.

\section{6 Institutionalising periodic, high-quality evaluations}

Several national evaluations have been undertaken, such as the NIPCCD Study (1992) and NCAER Study (2001). Yet, although ICDS is one of the most studied health and nutrition programmes, relatively few impact evaluations have drawn on the large samples and rigorous evaluation designs are needed to infer impact (Bredenkamp et al. 2005). To measure the true impact of the programme, there needs to be a better understanding of the importance of including data on treatment and comparison groups in the evaluation, and moving beyond bivariate analysis to employ more rigorous econometric techniques. Also, key stakeholders need to be engaged in the evaluation process so that the findings are more likely to be used in programme planning.

The most useful contribution to the evaluation of ICDS has come from the inclusion of questions related to the programme in the regular National Family Health Surveys (NFHS) since 2005/06. It is now possible to relate access to ICDS services to a range of health behaviours and health status indicators across India. However, the NFHS is only conducted once every six to eight years and is not representative at the district level. The District Level Household Surveys (DLHS) can provide district level data on nutritional outcomes, but does not have programme-specific indicators.

A few large-scale household surveys in recent years have focused on nutrition and ICDS. The 'Focus On Children Under Six (FOCUS)' report published by the Citizen's Initiative for the Rights of Children Under Six (CIRCUS 2006) surveyed 122 villages and generated tremendous popular interest in the effectiveness of ICDS. Some international organisations that provide large-scale support to ICDS, such as CAREIndia, conduct population-based surveys at regular intervals, especially on the operational elements of the programme. World Bank support to the ICDS programme (ICDS-III/WCD Project 1999/2006) provided the first large sample ICDSspecific impact evaluation with a pre- and postintervention evaluation design.

Still, these ad hoc surveys are insufficient to provide a complete picture of the performance of a nationwide programme. What is critical is to institutionalise regular, independent assessments of the key programme outputs/outcomes, especially at the decentralised levels.

\section{Recent steps towards an outcome-oriented approach}

The challenges are immense, but in recent years there have been some noteworthy steps forward. Institutions, interventions and measures that reflect an increased emphasis on results have begun to emerge and evolve:

1 Revision of the MIS: Following consultation with government and development partners, the MWCD recently revised and reduced the number of records and registers, as well as the MIS indicators. The objective was to make recording and reporting formats more relevant, less burdensome and easier to use. Also, ICDS officials are now required to prepare progress reports based on the analysis of key performance monitoring indicators.

\section{Engagement of professional and technical} organisations: A Central Monitoring Unit at the National Institute of Public Cooperation and Child Development (NIPCCD) is being piloted to monitor and assess the effectiveness of ICDS implementation. It cooperates with several state-based medical colleges and home science institutions.

3 Use of information technology to improve programme monitoring: At least five states have piloted different computer-based monitoring information systems, such as the web-enabled MIS application in Maharashtra. Success has been mixed since most of these have not yet reached their potential as tools to improve performance.

4 Introduction of field-based performance-management toolkits: CARE's Integrated Nutrition and Health Project (INHP-II) introduced performance-management tools in nine project states. These included guidelines to 
help Supervisors facilitate sector meetings, manuals to help AWWs plan and conduct home visits, and checklists for supervising AWCs.

5 Introduction of new WHO Child Growth Standards: Last year, the MWCD adopted the new WHO child growth standards as the official measure of nutritional status. Previously, the MIS had used the growth standards of the Indian Academy of Paediatrics (IAP), which produced lower estimates of malnutrition than those produced using the NFHS, resulting in divergent views of programme performance.

6 Performance Rating System: An accreditation system whereby AWCs are graded on childrelated outcomes and quality standards is being introduced. Some States have piloted it with support from UNICEF and the MWCD is standardising the accreditation system to link performance to rewards.

7 Community monitoring: Pilots have been undertaken by civil society organisations with district officials to engage the community in monitoring AWC services. Social audits and the active engagement of panchayats in monitoring the timely opening of AWCs and quality of supplementary food have been tested in a few States.

\section{Recommendations}

The political environment is conducive to an outcome-oriented approach. Government commitment and an increased budgetary allocation to the ICDS programme during the Eleventh Plan, represents a window of opportunity to strengthen monitoring and evaluation within ICDS, as well as more broadly in the nutrition sector.

The challenge is to translate the Government's vision into action. The outcomes-oriented approach to ICDS should be institutionalised to facilitate, and mandate, the tracking of outcomes. Some concrete steps that could be taken at central and state levels include:

- Monitoring and evaluation needs to be prioritised by ICDS senior management. A strong system can improve programme effectiveness and achieve better results. National and state-specific strategies should be developed to strengthen the existing system by agreeing an implementation plan.

- An in-depth review of the data that is collected is needed to determine which indicators are the most important for programme management, at what level, at what frequency of collection and for which decisions. Only key indicators that are specific, measureable and attributable to ICDS should be chosen and systems put in place to ensure that the data is used to inform decision-making. A useful first step would be to develop an ICDS 'results framework' listing inputs, outputs and outcomes that ICDS will be held responsible for monitoring (Appendix 1).

- Once appropriate output and outcome indicators are identified, State, District and Block targets should be agreed and action plans developed, similar to those developed for Gol's flagship National Rural Health Mission. Progress should be measured regularly so that follow-up action can be taken.

- ICDS needs additional qualified staff at the State and District levels to work on programme monitoring. Currently, with the exception of a few States, there are no technically qualified personnel at the State level or below whose job descriptions focus solely on monitoring.

- District and Block officials need to be trained in data analysis, interpretation and use in programme implementation. Specific training modules on monitoring and data analysis are required.

- Annual surveys, rapid assessments, operations research, and social assessments need to be institutionalised to assess programme outputs, outcomes and impact. Such evaluations must be time-sensitive and provide detailed information regarding the effectiveness of specific processes/interventions during a defined reference period.

- Finally, all civil society organisations and development partners who contribute to shaping the national debate on malnutrition should consider how they can best support the government in strengthening the monitoring system to ensure that the ICDS programme delivers on outputs and outcomes. 


\section{Note}

* The authors gratefully acknowledge the generous advice of Ashi K. Kathuria, Meera Shekar, Mohini Kak, Sunil Babu, Sridhar Srikantiah and V. Ramesh Babu. The findings, interpretations and conclusions expressed in this article are entirely those of the authors

\section{References}

Bredenkamp, C.; Akin, J. and Gragnolati, M. (2005) 'Malnutrition and India's ICDS Program: Evidence of Program Impact?', unpublished manuscript

Citizens' Initiative for the Rights of Children Under Six (CIRCUS) (2006) Focus on Children Under Six, Abridged Report

Gragnolati, Michele; Bredenkamp, Caryn; Das Gupta, Monica; Yi-Kyoung, Lee and Shekar, Meera (2006) 'ICDS and Persistent Undernutrition - Strategies to Enhance the Impact', Economic and Political Weekly, 25 March: 1193-200

Kusek, Jody Zall and Rist, Ray C. (2004) Ten Steps to a Results-based Monitoring and Evaluation System: A Handbook for Development Practitioners, Washington DC: World Bank

Ministry of Finance (MoF), Government of India (2005) Outcome Budget 2005-06, http://finmin.nic.in/reports (accessed 15 May 2009)

Ministry of Women and Child Development (MWCD) (2009) ICDS Quarterly Progress Report, 30 September 2008

Ministry of Women and Child Development (MWCD) (2006) Sub-Group Report on ICDS and should not be attributed in any manner to the Ministry of Women and Child Development, the Government of India, or the World Bank, its affiliated organisations or members of its Board of Executive Directors or the countries they represent.

and Nutrition in Working Group Report on Development of Children for the Eleventh Five Year Plan (2007-2012) Vol. II, http://wcd.nic.in/ (accessed 15 May 2009)

National Council of Applied Economic Research (NCAER) (2001) Concurrent Evaluation of ICDS (1998-1999)

National Institute of Public Cooperation and Child Development (NIPCCD) (2006) Syllabus for Job Training of Anganwadi Workers/Supervisors/ CDPOs/ACDPOs

National Institute of Public Cooperation and Child Development (NIPCCD) (1992) National Evaluation of ICDS Scheme

Planning Commission, Government of India (2008) Inclusive Growth: Vision and Strategy in Eleventh Five Year Plan (2007-12) Vol I, New Delhi: Oxford University Press, www.planningcommission.nic.in/plans/planrel/ fiveyr/default.html (accessed 15 May 2009)

Shekar, Meera; Heaver, R. and Yi-Kyoung Lee (2006) Repositioning Nutrition as Central to Development: A Strategy for Large Scale Action, Washington DC: World Bank

UNICEF (1998) The State of the World's Children 1998: Focus on Nutrition, New York: Oxford University Press 


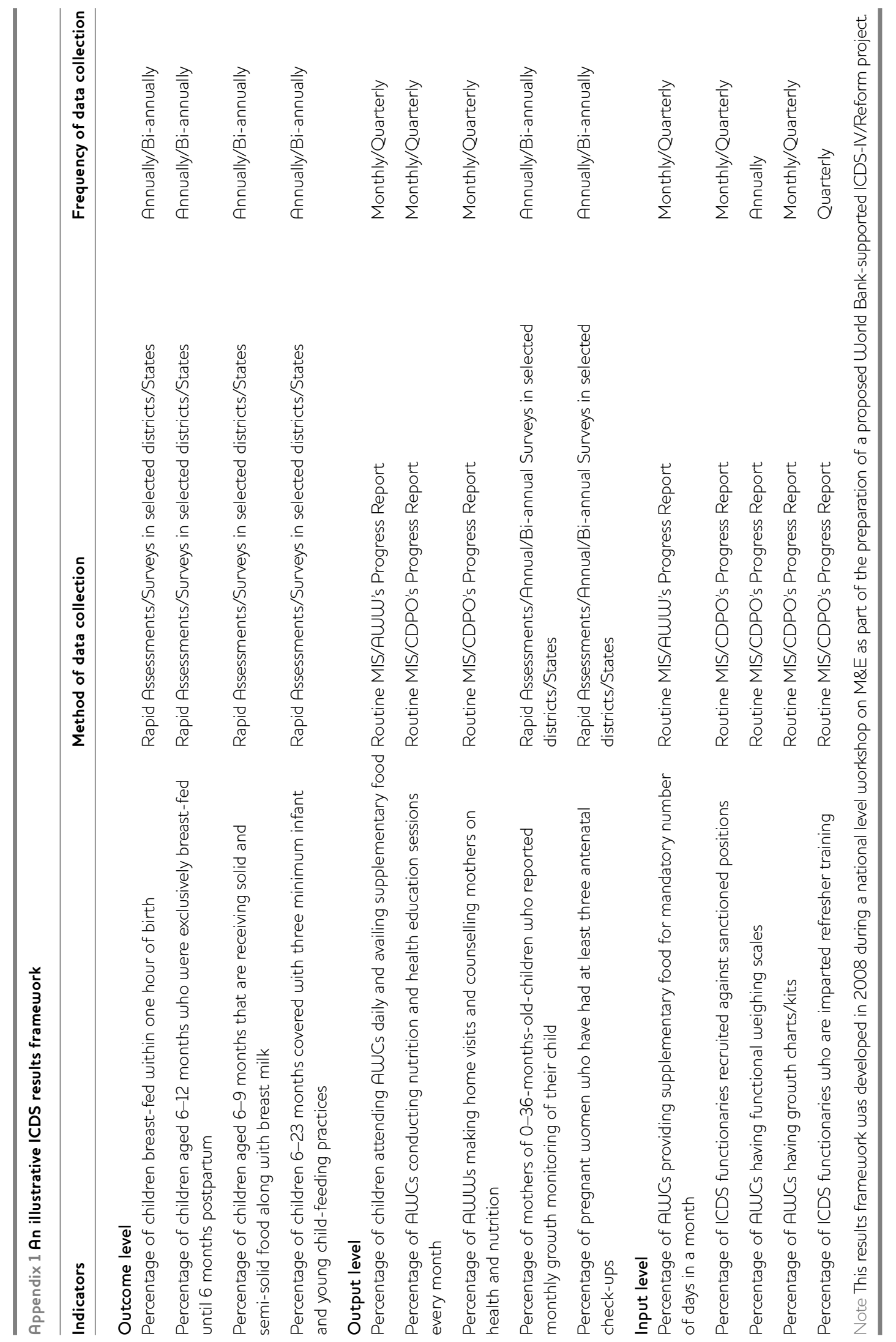

IDS Bulletin Volume 40 Number 4 July 2009 